\title{
Efficacy and Safety of Thermal Ablation Techniques for the Treatment of Primary Papillary Thyroid Microcarcinoma: A Systematic Review and Meta-Analysis
}

\author{
Yangsean Choi ${ }^{1}$ and So-Lyung Jung ${ }^{2}$
}

\begin{abstract}
Background: The increased incidence of primary papillary thyroid microcarcinoma (PTMC) has led to increased research in the field of nonsurgical therapeutic options for those who refuse surgery or are at high risk for surgery. The study aimed at comprehensively evaluating the efficacy and safety of thermal ablation techniques for the treatment of PTMC via a systematic review and meta-analysis.
\end{abstract}

Methods: The Pubmed MEDLINE and EMBASE databases were searched for studies reporting the efficacy and safety of thermal ablations (radiofrequency-, laser-, and microwave-ablations [RFA, LA, and MWA]) until August 10, 2019. A review of 105 potential papers identified 11 eligible papers, including 715 patients. The pooled proportions of complete disappearance and recurrence, and the pooled estimates of mean volume reduction and its rate of the treated PTMC were assessed by using random-effects modeling. The pooled proportions of overall and major complications were calculated. Subgroup analysis was performed according to the treatment modality. Between-study heterogeneity was explored by using $\chi^{2}$ statistic for pooled estimates and inconsistency index $I^{2}$. Quality of the studies was evaluated by using the Risk of Bias Assessment Tool for Nonrandomized Studies.

Results: The pooled proportions of complete disappearance and recurrence of PTMC were $57.6 \%$ [95\% confidence interval (CI): $35.4-79.8$ ] and $0.4 \%$ [95\% CI: 0-1.1], respectively. The pooled estimates of mean volume reduction and its rate were $73.5 \mathrm{~mm}^{3}$ [52.4-94.6 $\mathrm{mm}^{3}$ ] and $98.1 \%$ [95\% CI: 96.7-99.5], respectively. The pooled proportions of overall and major complications were 3.2\% [95\% CI: 1.1-5.2] and 0.7\% [95\% CI: 0-1.5], respectively. Significant between-study heterogeneity was observed for complete disappearance $\left(p<0.001, I^{2}: 99 \%\right)$, mean volume reduction $\left(p<0.001, I^{2}: 93 \%\right)$, and its rate $\left(p<0.001, I^{2}: 86 \%\right)$. Subgroup analysis revealed heterogeneity of the complete disappearance proportion among the treatment modality ( $I^{2}$ range: $\left.95-100 \%\right)$. RFA showed the highest mean volume reduction rate $(99.3 \%)$, followed by MWA $(95.3 \%)$ and LA $(88.6 \%)(p<0.001)$.

Conclusions: All thermal ablation techniques were effective and safe for the treatment of PTMC. However, each treatment modality had significant heterogeneity with respect to complete disappearance of PTMC. Compared with RFA and MWA, LA was less effective in reducing the volume of PTMC.

Keywords: papillary thyroid microcarcinoma, radiofrequency ablation, laser ablation, microwave ablation, thermal ablation, meta-analysis

\section{Introduction}

A MONG THE SUBTYPES of thyroid carcinomas, papillary thyroid carcinoma is the most common, accounting for more than $70 \%$ of the thyroid malignancies (1). Papillary thyroid microcarcinoma (PTMC) is defined as papillary thyroid carcinoma measuring less than $1.0 \mathrm{~cm}$ in size and has very favorable prognosis, with a disease-specific mortality rate of less than $1 \%(2,3)$. Among the treatment options for PTMC, surgical management is the first-line treatment
(4). However, it is not suitable for patients for thyroidectomy may be high-risk surgery or for those refusing surgery (5). Active surveillance is also an alternative management approach recommended by the American Thyroid Association (4); however, there is a risk of locoregional metastasis $(2-6 \%)(2,3)$.

Accumulating evidence suggests that thermal ablation techniques, including radiofrequency ablation (RFA), microwave ablation (MWA), and laser ablation (LA), are effective and safe treatment modalities for both benign thyroid

\footnotetext{
${ }^{1}$ Department of Radiology, Seoul St. Mary's Hospital, College of Medicine, The Catholic University of Korea, Seoul, Korea.

${ }^{2}$ Department of Radiology, Yeouido St. Mary's Hospital, College of Medicine, The Catholic University of Korea, Seoul, Korea.
} 
nodules (6-9) and recurrent thyroid cancers (10-13). Compared with surgical treatment, similar efficacy and lower rates of complications have been reported for RFA $(10,12)$ and MWA (14) for the treatment of recurrent papillary thyroid carcinoma and primary PTMC, respectively.

Recent studies have reported that thermal ablations are effective and safe for treating primary PTMC (14-25). However, there are still concerns and comprehensive assessment of thermal ablation techniques in terms of the treatment efficacy and safety of PTMC is necessary to optimize personalized care in the face of uncertain evidence and to bridge the evidence gaps in the future. Therefore, this study aimed at comprehensively evaluating the efficacy and safety of thermal ablation techniques for the treatment of PTMC. To the best of our knowledge, this is the first systematic review and meta-analysis evaluating the efficacy and safety of different thermal ablations for the treatment of PTMC.

\section{Materials and Methods}

\section{Literature search strategy}

This meta-analysis was performed according to the Preferred Reporting Items for a Systematic Review and Metaanalysis (PRISMA) guidelines (26). The PubMed, MEDLINE, and EMBASE databases were searched to identify publications on thermal ablation in treating PTMC. No limitation was placed on the beginning date of literature, and the search was finalized on August 10, 2019. The following search terms were used: [(thyroid) AND (microcarcinoma OR micropapillary) AND (carcinoma OR cancer OR malignancy) AND (radiofrequency ablation OR RFA OR LA OR laser ablation OR microwave ablation OR thermal ablation)]. The bibliographies of the selected articles were further reviewed to search for other relevant articles. Two reviewers (blinded and blinded) independently performed the literature search; any disagreement in terms of inclusion was resolved by discussion until unanimity was attained by all authors. We were not blinded to authors, institutions, journals, or interventions while selecting studies or extracting data.

\section{Inclusion and exclusion criteria}

Studies investigating thermal ablation techniques (i.e., RFA, LA, and MWA) for the treatment of PTMC were eligible for inclusion. The included studies met the following criteria: (1) population and intervention: Patients were diagnosed with primary PTMC; the main treatment modality was either RFA, LA, or MWA; and sample size was $\geq 5$ patients. (2) Study design: Both retrospective and prospective observational studies were included. (3) Outcomes: Studies reported details on treatment outcomes (i.e., volume reduction, complete disappearance, recurrence of nodules) and complications.

Any study meeting one of the following exclusion criteria was excluded: (1) case reports or series with $<5$ patients; (2) abstracts, reviews, comments, and editorials; (3) articles not written in English; (4) studies with partially overlapping cohorts. Two reviewers (blinded and blinded) independently reviewed the literature in consensus.

\section{Data extraction}

From the eligible studies, the following data were extracted into standardized formats: (a) study characteristics: authors, year of publication, affiliation, duration of study, study design, and sample size; (b) patients' demographic and clinical characteristics: age, sex, ablation technique (RFA, MWA, or LA), nodule characteristics (size and volume), operators' experience, and follow-up duration; (c) outcomes: mean volume reduction $\left(\mathrm{mm}^{3}\right)$ of nodules between treatment sessions, volume reduction rate $(\%)$ calculated as [(initial volume - final volume) $\times 100 /$ initial volume], complete disappearance (determined on the final follow-up ultrasound [US] scan), recurrence (determined pathologically), and volume reduction rate; and (d) major and minor complications per patient. One reviewer (blinded) extracted the data, and another reviewer (blinded) confirmed the data's validity.

\section{Quality assessment}

The quality of the included studies was then independently assessed in consensus by two authors (blinded and blinded) by using the structured criteria of the Risk of Bias Assessment Tool for Non-randomized Studies (RoBANS) (27).

\section{Definitions of complications}

Definitions of major and minor complications were based on definitions by the Society of Interventional Radiology $(28,29)$. A major complication was defined as one that might be life-threatening, result in substantial morbidity or disability, or prolong hospitalization if left untreated. In this study, major complications included transient (lasting $>1$ month) or permanent voice changes and hypothyroidism. Minor complications included immediate hoarseness (lasting $<1$ week), hematoma, and moderate pain requiring medication to relieve it. Side effects-not requiring therapy or prescription medication-included mild, transient postprocedural pain, heat sensation, and neck discomfort.

\section{Data synthesis for assessing the pooled proportions and estimates of treatment efficacy and complications}

The primary outcomes of this meta-analysis were the pooled proportions of complete disappearance of nodule, recurrence of nodule, overall complications and major complications, and pooled estimates of mean volume reduction rates of nodules. Meta-analytic pooling was performed by using the inverse variance method to calculate weights. Pooled proportions with 95\% confidence intervals (CIs) were obtained with the aid of Der Simonian-Laird random-effects modeling (30-32). Subgroup meta-regression analysis was performed by the treatment modality (RFA, MWA, and LA).

Between-study heterogeneity was calculated by the $\chi^{2}$ statistic for pooled estimates $(p<0.05$, indicating significant heterogeneity) and the Higgins inconsistency index $I^{2}(0-40 \%$, may have insignificant heterogeneity; 30-60\%, 50-90\%, and 75-100\%, may have moderate, substantial, and considerable heterogeneity, respectively) (33). Publication bias was evaluated by using funnel plots and Egger's test, with a $p$-value $<0.1$ indicating significant bias (34). Publication bias-adjusted pooled proportions and estimates were also calculated via the trim-and-fill method (35), where agreement between the unadjusted and adjusted pooled proportions and estimates 
indicates minimal publication bias. All statistical metaanalyses were performed by one author (blinded) using the "meta" and "metafor" packages in R statistical and computing software (version 3.5.1, Vienna, Austria).

\section{Results}

\section{Literature search}

The selection process of studies from the literature search is depicted in Figure 1. The initial literature search identified 105 articles. After removal of duplicates, 90 were screened for eligibility. Of these, 77 were excluded after reviewing the abstracts (20 review articles, 1 case report, 4 letters/editorials/abstracts, 42 articles of irrelevant contents, and 10 nonEnglish articles). The full texts of the remaining 13 articles were then thoroughly reviewed; two articles were further excluded due to a small sample size ( $<5$ patients) (36) and having partially overlapping cohorts (15). Between the two studies with overlapping cohorts, the study with a larger sample size was chosen. Finally, 11 articles with a total of 715 patients with 748 primary PTMC were included (1420,22-25). No additional eligible study was identified after a search of bibliographies within these articles.

\section{Characteristics of included studies}

The detailed characteristics of the 11 eligible studies are shown in Table 1. Among the 11 original articles included, there were 7 retrospective studies $(14,15,17-20,25), 2$ prospective studies $(22,24)$, and 2 studies with unclear study design $(16,23)$. All included studies were carried out in single centers. The cohort sizes ranged from 6 to 185 patients, with numbers of PTMC ranging from 6 to 206 total nodules. The mean ages of included studies' patients ranged from 42.2 to 59.5 years. The mean largest diameters of PTMC ranged from 1.8 to $7.3 \mathrm{~mm}$. Nine of 11 studies had a mean volume re- duction rate of more than $90 \%$ (range, 56.3-100\%). The mean follow-up periods of included studies ranged from 7.8 to 25.7 months, with six studies reporting more than 1 year of mean follow-up periods $(14,15,18-20,25)$. The type of thermal ablation techniques included four MWA $(14,18,22,23)$, four RFA $(16,17,20,24)$, and three LA $(15,19,25)$. The experience of operators was reported in seven studies (range, 220 years of experience). All study settings were based in Asian countries-10 in China (14-19,22-25) and 1 in Korea (20). A single thermal ablation session was performed in four studies $(14,15,20,22)$, while one study indicated a maximum of two LA sessions (19). Six studies did not describe the number of sessions performed (16-18,23-25). The quality assessment by RoBANS showed moderate overall quality of the included studies, with all studies satisfying at least five of the eight criteria (Fig. 2). All studies described the thermal ablation techniques and equipment clearly.

\section{Pooled estimates of mean volume reduction rates}

The pooled estimates of mean volume reduction and its rate were $73.5 \mathrm{~mm}^{3}$ (95\% CI: $\left.52.4-94.6 \mathrm{~mm}^{3}\right)$ and $98.1 \%$ (95\% CI: 96.7-99.5), respectively (Fig. 3A, B). There were significant heterogeneities for both (mean volume reduction, $I^{2}=93 \%$; mean volume reduction rate, $I^{2}=86 \%$; Cochran's Q method, both, $p<0.001)$. The funnel plot and Egger's test showed a significant publication bias (Fig. 4A, B, $p<0.001$ ). After the trim-and-fill method adjusting publication bias, the adjusted pooled estimates for mean volume reduction and its rate were $61.2 \mathrm{~mm}^{3}$ (95\% CI: $\left.48.8-73.7 \mathrm{~mm}^{3}\right)$ and $99.2 \%$ (95\% CI: 97.6-100), respectively.

\section{Pooled proportions of complete disappearance and recurrence of nodules}

Among 728 nodules, 415 nodules showed complete disappearance after treatment. Among eight studies reporting
FIG. 1. A flow diagram of PRISMA.

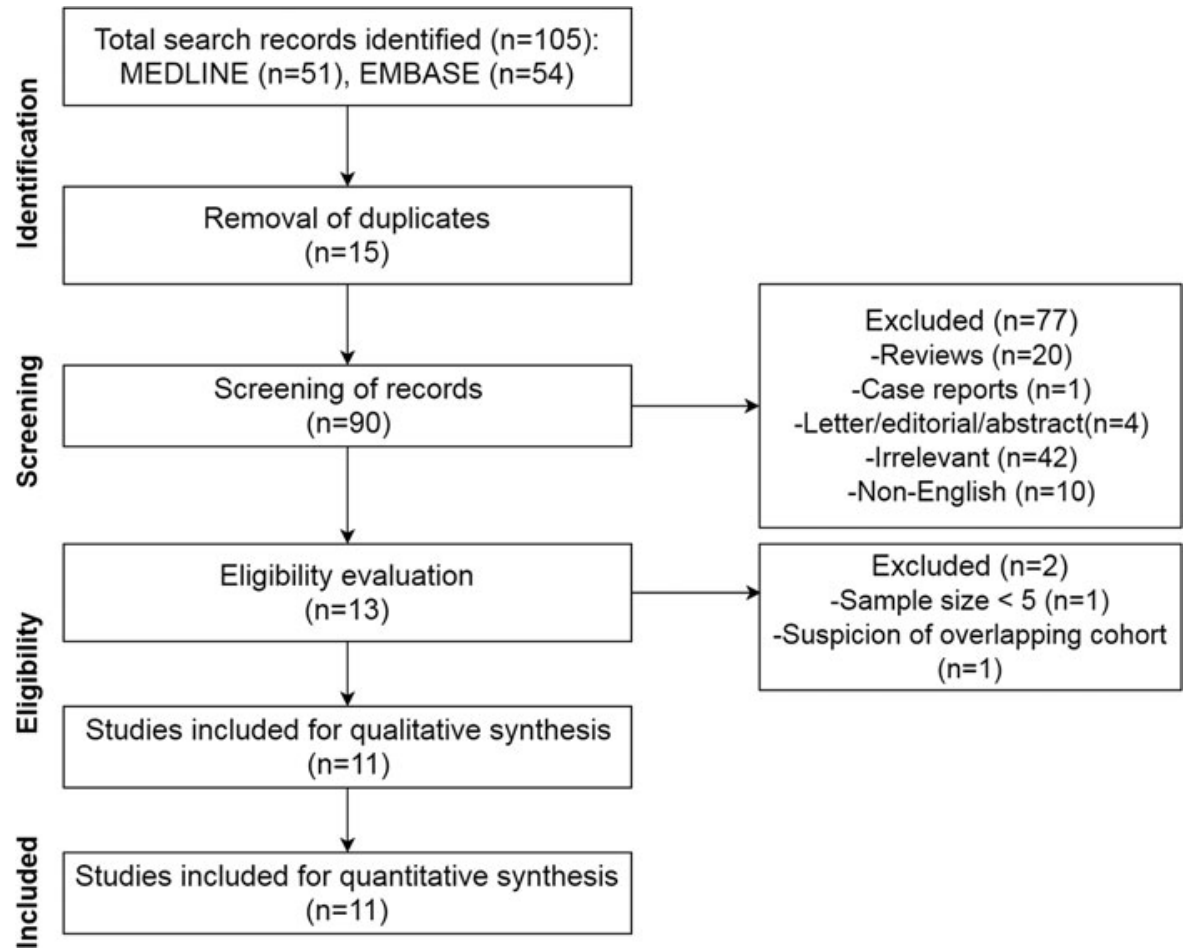




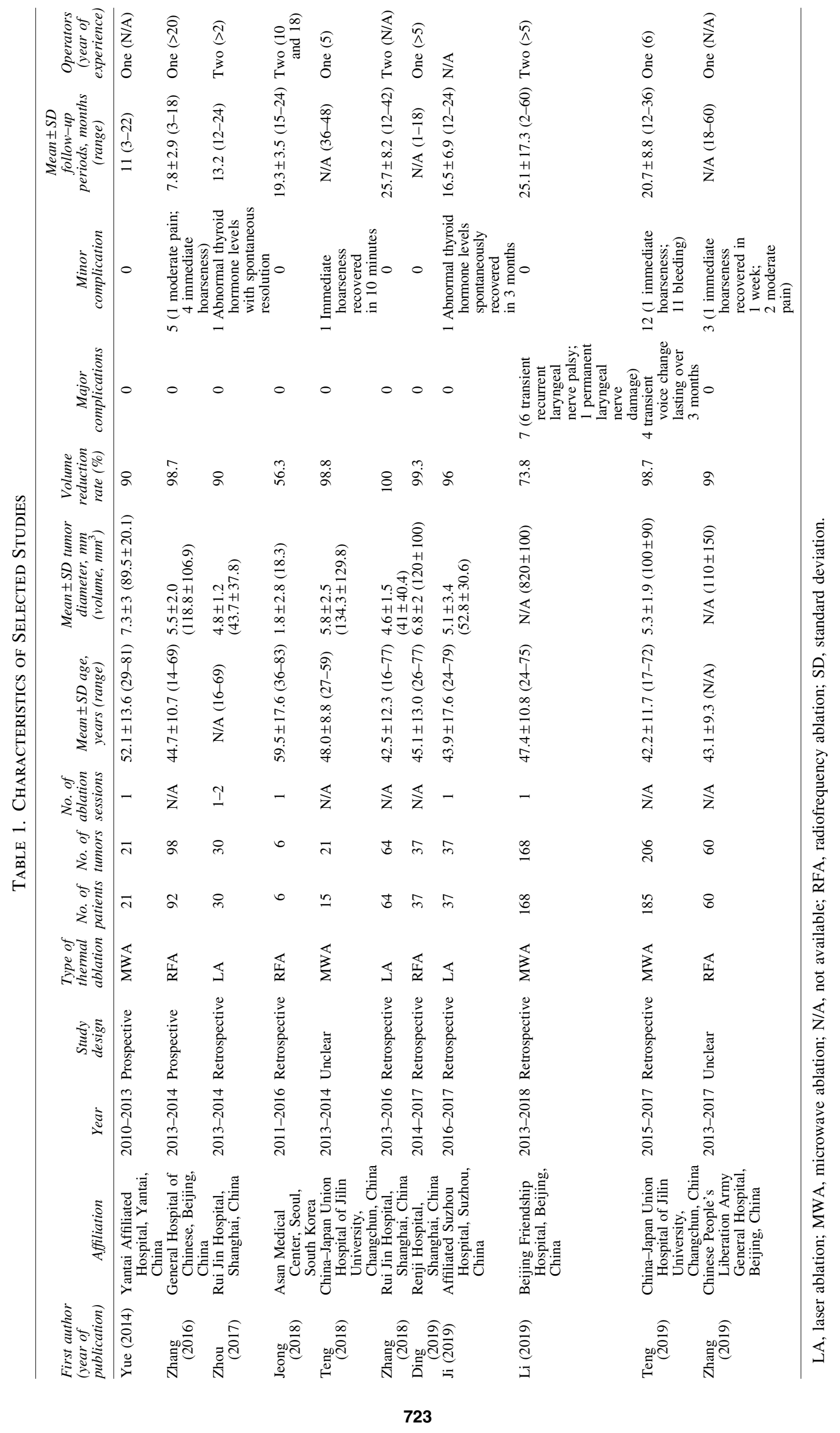




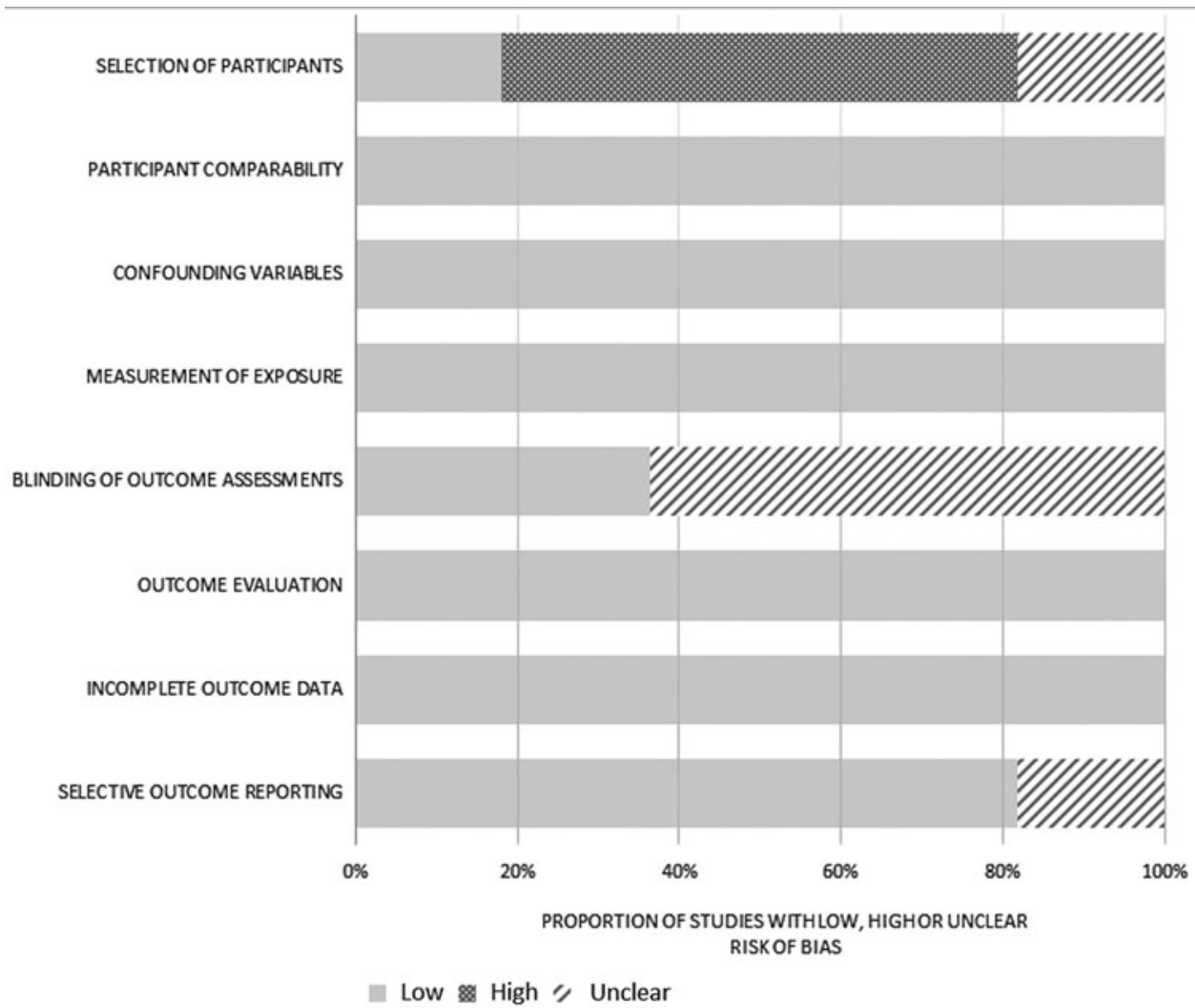

mean follow-up periods, the mean follow-up of completely disappeared PTCM $(n=298)$ was 21.1 months (range, 1125.7 months). The pooled proportion of complete disappearance was $57.6 \%$ (95\% CI: $35.4-79.8 ; I^{2}=99 \%$ ) (Table 2 and Fig. 3C). The funnel plot showed symmetric distribution of complete disappearances of nodules, and publication bias was not significant in Egger's test ( $p=0.273)$ (Fig. 4C). Significant heterogeneity was observed in the proportion of complete disappearance of nodules $(p<0.001)$. Nine recurrences among 728 nodules were reported in three studies $(14,15,18)$. The pooled proportion of recurrence was $0.4 \%$ (95\% CI: $0-1.1 ; I^{2}=0 \%$ ) (Table 2 and Fig. 3D). In both the funnel plot and Egger's test, significant publication bias was observed for recurrences $(p=0.055)$ (Fig. 4D). However, the trim-and-fill estimate of recurrence $(0.6 \%)$ was inconsistent with the pooled proportion. No heterogeneity was observed in the reported recurrence rate $(p=0.704)$.

\section{Pooled proportions of complications}

Thirty-four complications among 728 nodules in 715 patients were reported. The pooled proportion of major complications was $0.7 \%$ (95\% CI: $\left.0-1.5 ; I^{2}=0 \%\right)$ (Table 2 and Fig. 5A). The funnel plot showed asymmetric distribution of major complications, but publication bias was not significant in Egger's test $(p=0.199)$ (Fig. 6A). The pooled proportion of overall complications was 3.2\% (95\% CI: $1.1-$ $5.2 ; I^{2}=34 \%$ ) (Table 2 and Fig. 5B). In both the funnel plot and Egger's test, no significant publication bias was seen for overall complications $(p=0.247)$ (Fig. 6B). No heteroge- neity was noted in both overall $(p=0.123)$ and major $(p=0.8)$ complications. The trim-and-fill estimates of overall and major complications were consistent with pooled proportions, indicating little to no publication bias (Table 2).

\section{Details on major and minor complications}

All 11 major complications were voice changes-10 transient voice changes lasting for at least 1 month $(14,18)$ and 1 permanent voice change (14). Twenty-three minor complications were reported, including 3 instances of moderate pain, 2 abnormal thyroid hormone levels, 11 hematomas, and 7 instances of immediate hoarseness. All immediate hoarseness occurred soon after the completion of the procedure and resolved within 10 minutes to 1 week $(16,18,23,24)$. Other than the moderate pain relieved by medication, all minor complications resolved spontaneously within 3 months. The frequency of major and minor complications was $1.5 \%(11 / 715)$ and $3.2 \%$ (23/715), respectively.

\section{Subgroup analysis according to the treatment modality}

Subgroup analysis according to the treatment modality was performed for the treatment efficacy outcomes showing significant heterogeneity (i.e., complete disappearance and mean volume reduction rate) (Table 3 ). There were no significant differences among the treatment modalities for complete disappearance of nodules $(p=0.351)$. However, all modalities showed large heterogeneity $\left(I^{2}: 95-100 \%\right)$. 
A study

Yue (2014)

Zhang (2016)

Zhou (2017)

Jeong (2018)

Teng (2018)

Zhang (2018)

Ding (2019)

Ji (2019)

Li (2019)

Teng (2019)

Zhang (2019)

Random effects model

Yue (2014)

Zhang (2016)

Zhou (2017)

Jeong (2018)

Teng (2018)

Zhang (2018)

Ding (2019)

Ji (2019)

Li (2019)

Teng (2019)

Zhang (2019)

Random effects model

Mean volume reduction rate

$95 \% \mathrm{Cl}$

90.000 [84.012; 95.988]

96.000 [77.609; 114.391]

$79.200[56.191 ; 102.209]$

56.300 [16.292; 96.308]

98.800 [95.966; 101.634]

$95.600[75.167 ; 116.033]$

$99.300[98.172 ; 100.428]$

$89.200[63.809 ; 114.591]$

73.800 [64.757; 82.843]

98.700 [98.181; 99.219]

$99.900[99.773 ; 100.027]$

98.065 [96.668; 99.462]

$$
0
$$

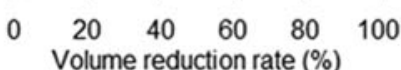

Volume reduction rate $(\%)$

\section{Study}

Yue (2014)

Zhang (2016)

Zhou (2017)

Jeong (2018)

Teng (2018)

Zhang (2018)

Ding (2019)

Ji (2019)

$\mathrm{Li}(2019)$

Teng (2019)

Zhang (2019)

Random effects model

Complete disappearance $\quad 95 \% \mathrm{Cl}$

$0.222[0.030 ; 0.414]$

$0.102[0.042 ; 0.162]$

$0.333[0.165 ; 0.502]$

$0.500[0.100 ; 0.900$

$0.952[0.861 ; 1.000]$

$0.797[0.698 ; 0.895]$

$0.974[0.923 ; 1.000]$

$0.324[0.173 ; 0.475]$

$0.227[0.160 ; 0.294$

$0.845[0.795 ; 0.894]$

$1.000[0.977 ; 1.000]$

$0.576[0.354 ; 0.798]$

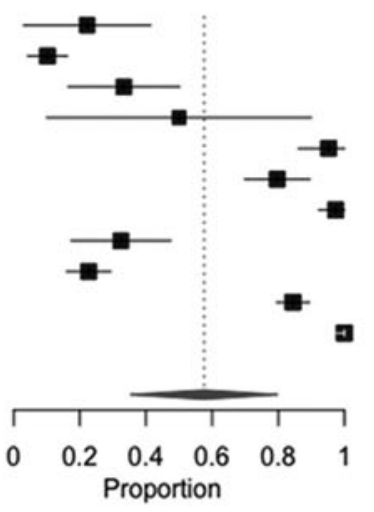

D Study

Yue (2014)

Zhang (2016)

Zhou (2017)

Jeong (2018)

Teng (2018)

Zhang (2018)

Ding (2019)

Ji (2019)

Li (2019)

Teng (2019)

Zhang (2019)

Random effects model

\section{Recurrence $\quad 95 \% \mathrm{Cl}$}

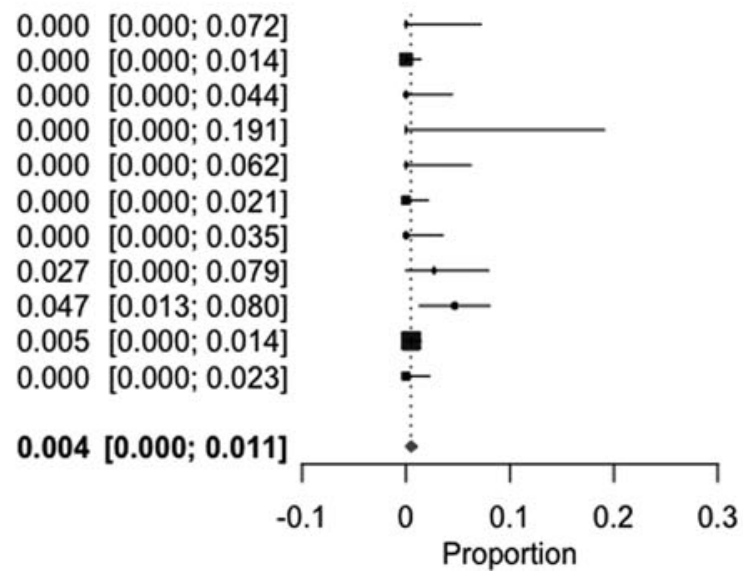

FIG. 3. Forest plots of pooled estimates of $(\mathbf{A})$ mean volume reduction $\left(\mathrm{mm}^{3}\right)$, (B) mean volume reduction rate $(\%)$, and pooled proportions of $(\mathbf{C})$ complete disappearance and $(\mathbf{D})$ recurrence of PTMC. PTMC, papillary thyroid microcarcinoma. 

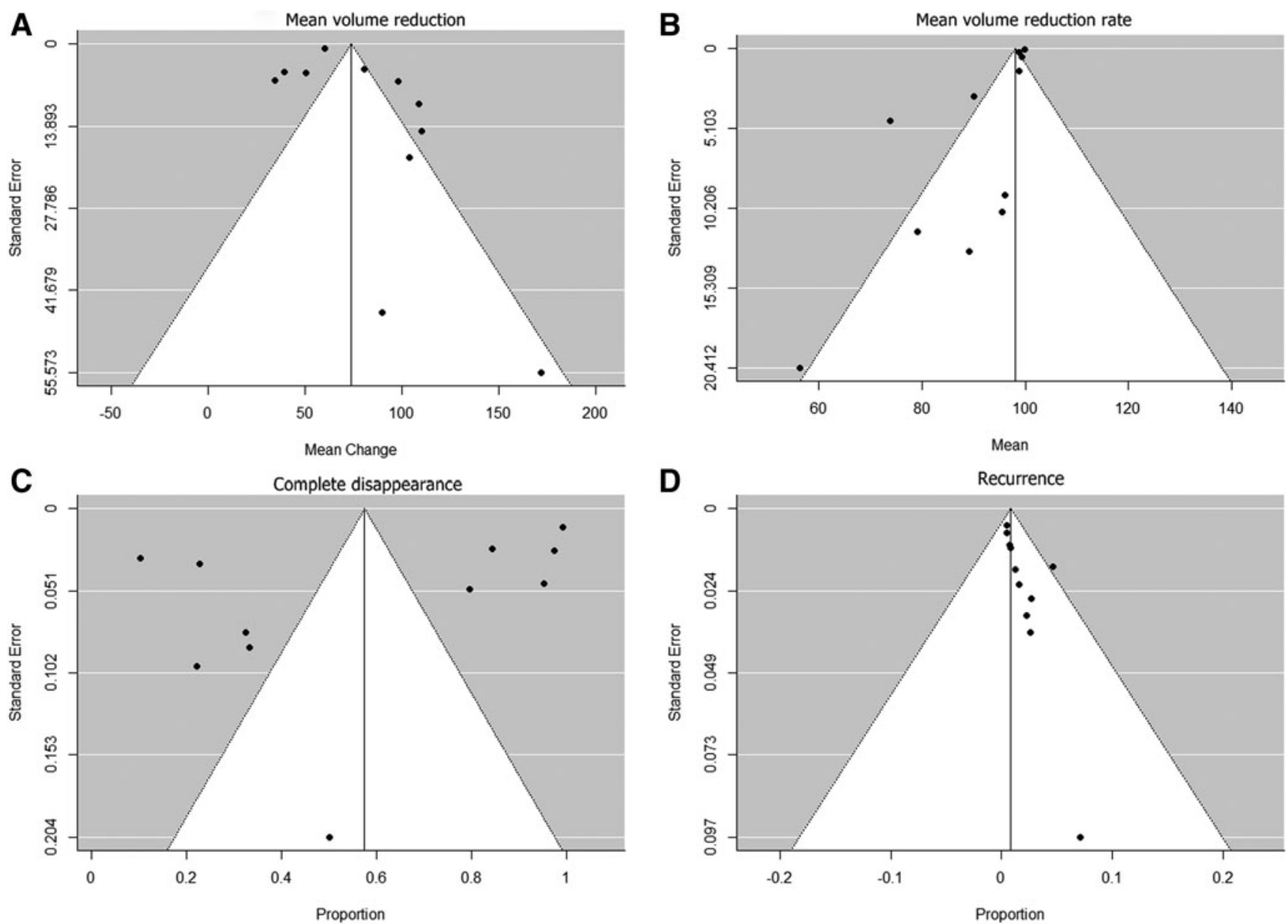

FIG. 4. Funnel plots of (A) mean volume reduction, $(\mathbf{B})$ mean volume reduction rate, $(\mathbf{C})$ complete disappearance, and (D) recurrence of PTMC. Asymmetrically distributed dots (i.e., studies) from the midline (A, B, D) indicate the possibility of publication bias.

Table 2. Summary Estimates of the Meta-Analytic Pooled Proportions and Estimates for Efficacy and Safety of Thermal Ablations

\begin{tabular}{|c|c|c|c|c|c|c|}
\hline \multirow[b]{2}{*}{ Complications } & \multicolumn{3}{|c|}{ Summary estimate } & \multirow[b]{2}{*}{$\begin{array}{c}\text { p-Value for } \\
\text { publication } \\
\text { bias }^{\mathrm{c}}\end{array}$} & \multicolumn{2}{|c|}{ Trim-and-fill estimate } \\
\hline & $\begin{array}{c}\text { Pooled proportions } \\
{[95 \% \mathrm{CI}]}\end{array}$ & $\begin{array}{l}\mathrm{p} \text {-Value for } \\
\text { heterogeneity }^{\mathrm{a}}\end{array}$ & $\begin{array}{l}I^{2 \mathrm{~b}} \\
(\%)\end{array}$ & & $\begin{array}{l}\text { No. of } \\
\text { missing } \\
\text { studies }\end{array}$ & $\begin{array}{c}\text { Adjusted pooled } \\
\text { proportions } \\
{[95 \% \text { CI] }}\end{array}$ \\
\hline Overall & $3.2 \%[1.1-5.2 \%]$ & 0.123 & 34 & 0.247 & 5 & $2.1 \%[0-4 \%]$ \\
\hline Major & $0.7 \%[0-1.5 \%]$ & 0.8 & 0 & 0.199 & 5 & $0.8 \%[0-1.5 \%]$ \\
\hline \multicolumn{7}{|l|}{ Treatment efficacy } \\
\hline Complete disappearance & $57.6 \%[35.4-79.8 \%]$ & $<0.0001$ & 99 & 0.273 & 0 & $57.5 \%[35.5-79.5 \%]$ \\
\hline Recurrence & $0.4 \%[0-1.1 \%]$ & 0.704 & 0 & 0.055 & 6 & $0.6 \%[0-1.2 \%]$ \\
\hline Mean volume reduction $\left(\mathrm{mm}^{3}\right)$ & $73.5[52.4-94.6]^{\mathrm{d}}$ & $<0.0001$ & 93 & $<0.001$ & 4 & $61.2[48.8-73.7]$ \\
\hline Mean volume reduction rate $\mathrm{e}^{\mathrm{e}}$ & $98.1 \%$ [96.7-99.5\%] & $<0.0001$ & 86 & $<0.001$ & 5 & $99.2 \%[97.6-100 \%]$ \\
\hline
\end{tabular}

${ }^{a} p$-Value by the Cochran's $\mathrm{Q}$ method to test the heterogeneity of the pooled data $(p<0.05$ indicating significant heterogeneity).

'Higgins' index for heterogeneity (0-40\% may have insignificant heterogeneity; 30-60\%, 50-90\%, and 75-100\% may indicate moderate, substantial, and considerable heterogeneity, respectively).

'Egger's test ( $p<0.10$ indicating significant publication bias).

${ }^{\mathrm{d}}$ Pooled estimates for mean volume reduction with $95 \%$ CI.

${ }^{\mathrm{e}}$ Pooled estimates for mean volume reduction rate calculated as [(initial volume - final volume $) \times 100 /$ initial volume].

CI, confidence interval. 


\section{A study}

Yue (2014)

Zhang (2016)

Zhou (2017)

Teng (2018)

Zhang (2018)

Ding (2019)

Ji (2019)

Li (2019)

Teng (2019)

Zhang (2019)

Random effects model
Jeong (2018)

Major Complications $\quad 95 \% \mathrm{Cl}$

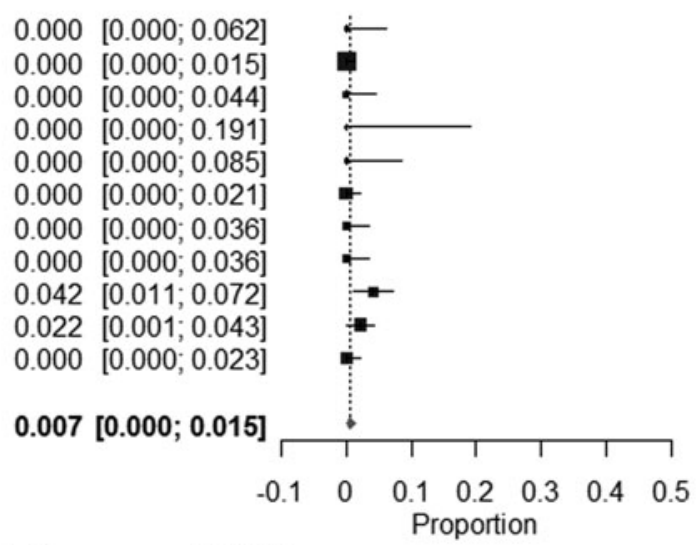

B Study

Yue (2014)
Zhang (2016)
Zhou (2017)
Jeong (2018)
Teng (2018)
Zhang (2018)
Ding (2019)
Ji (2019)
Li (2019)
Teng (2019)
Zhang (2019)

Random effects model
Overall Complications $\quad 95 \% \mathrm{Cl}$

$0.000[0.000 ; 0.062]$

$0.054[0.008 ; 0.101]$

$0.033[0.000 ; 0.098]$

$0.000[0.000 ; 0.191]$

$0.067[0.000 ; 0.193]$

$0.000[0.000 ; 0.021]$

$0.000[0.000 ; 0.036]$

$0.027[0.000 ; 0.079]$

$0.042[0.011 ; 0.072]$

$0.086[0.046 ; 0.127]$

$0.050[0.000 ; 0.105]$

$0.032[0.011 ; 0.052]$
FIG. 5. Forest plots of pooled proportions of (A) major and (B) overall complications of thermal ablation.
For the mean volume reduction rate, RFA showed the highest volume reduction rate $(99.3 \%)$ followed by MWA (95.3\%) and LA (88.6\%), and the differences were statistically significant $(p<0.001)$. However, no significant differences were found among the treatment modalities in the subgroup analysis of overall $(p=0.286)$ and major ( $p=0.406)$ complications (Table 4$)$.

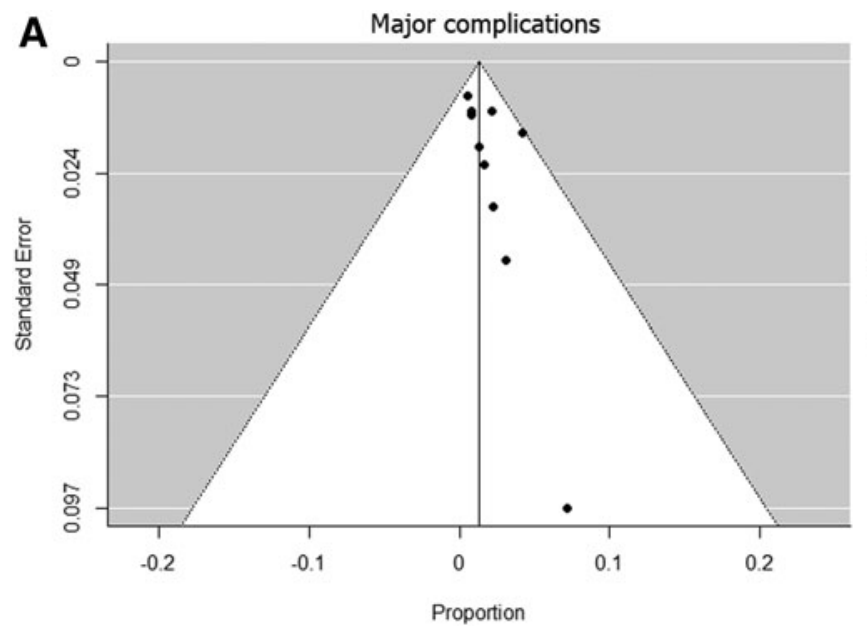

\section{Discussion}

In the current systemic review and meta-analysis evaluating the efficacy and safety of thermal ablation techniques (i.e., RFA, MWA, LA), treatment efficacy was favorable, as noted by the pooled proportion of complete disappearance of PTMC (57.6\% [95\% CI: 35.4-79.8]) and that of recurrence

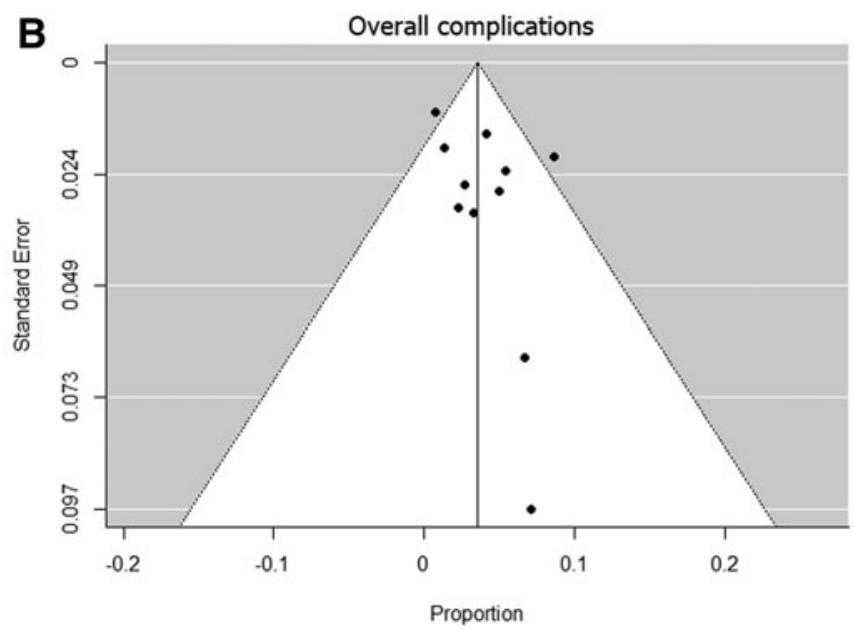

FIG. 6. Funnel plots for assessing publication bias of (A) major and (B) overall complications of thermal ablation. Slightly asymmetrical distribution of dots (i.e., studies) from the midline may indicate the possibility of publication bias; however, asymmetry was not statistically significant on Egger's test $(p>0.1)$. 
Table 3. Subgroup Analysis of Complete Disappearance, Mean Volume Reduction, and Mean Volume Reduction Rate of Nodules According to the Treatment Modality

\begin{tabular}{|c|c|c|c|c|c|c|c|c|c|}
\hline \multirow[b]{2}{*}{$\begin{array}{l}\text { Treatment } \\
\text { modality }\end{array}$} & \multicolumn{2}{|c|}{ Complete disappearance } & \multirow[b]{2}{*}{$\mathrm{p}^{\mathrm{b}}$} & \multicolumn{2}{|c|}{$\begin{array}{c}\text { Mean volume } \\
\text { reduction }\left(\mathrm{mm}^{3}\right)\end{array}$} & \multicolumn{3}{|c|}{$\begin{array}{l}\text { Mean volume } \\
\text { reduction rate }(\%)\end{array}$} & \multirow[b]{2}{*}{$\mathrm{p}^{\mathrm{b}}$} \\
\hline & $\begin{array}{c}\text { Pooled } \\
\text { proportions } \\
{[95 \% \mathrm{CI}]}\end{array}$ & $\mathrm{I}^{2 \mathrm{a}}(\%)$ & & $\begin{array}{c}\text { Pooled estimates } \\
\text { of mean volume } \\
\text { reduction } \\
{[95 \% \text { CI }]}\end{array}$ & $\mathrm{I}^{2 \mathrm{a}}(\%)$ & $\mathrm{p}^{\mathrm{b}}$ & $\begin{array}{c}\text { Pooled estimates } \\
\text { of mean volume } \\
\text { reduction rate } \\
{[95 \% \text { CI] }}\end{array}$ & $\mathrm{I}^{2 \mathrm{a}}(\%)$ & \\
\hline LA & $48.7 \%[2.1-95.4]$ & 95 & 0.351 & 41.7 [22.9-60.6] & 52 & 0.134 & $88.6 \%$ [75.2-101.9] & 0 & $<0.001$ \\
\hline MWA & $56.5 \%[16.3-96.7]$ & 99 & & $81[62.8-99.1]$ & 94 & & $95.3 \%[92.3-98.2]$ & 92 & \\
\hline RFA & $65.2 \%[24.3-100]$ & 100 & & $107.2[83-131.5]$ & 0 & & $99.3 \%$ [96.2-102.3] & 48 & \\
\hline
\end{tabular}

${ }^{\text {a}}$ Higgins' index for heterogeneity (0-40\%, may have insignificant heterogeneity; 30-60\%, 50-90\%, and 75-100\% may indicate moderate, substantial, and considerable heterogeneity, respectively).

${ }^{\mathrm{b}}$ Egger's test for publication and reporting bias $(p<0.10$ indicating significant publication bias).

${ }^{\mathrm{c}}$ Calculated as [(initial volume - final volume $) \times 100 /$ initial volume $]$.

LA, laser ablation; MWA, microwave ablation; RFA, radiofrequency ablation.

(0.4\% [95\% CI: 0-1.1]). The pooled estimate for mean volume reduction rate of PTMC after thermal ablation was 98.1\% [95\% CI: 96.7-99.5]. In addition, the pooled proportion of major complications was very low $(0.7 \%$ [95\% CI: $0-1.5]$ ), and all of them were non-life-threatening voice changes. Based on these results, all three thermal ablation techniques demonstrated efficacy and safety for the treatment of PTMC.

In subgroup analysis by treatment modality, the mean volume reduction rate of PTMC was comparable between RFA $(99.3 \%)$ and MWA $(95.3 \%)$, whereas that of LA was lower $(88.6 \%)$. This finding is consistent with a previous study by Korkusuz et al., who found comparable volume reductions between RFA and MWA in the treatment of benign thyroid nodules (37). Further, the relatively larger volume reduction of RFA compared with LA is consistent with a previous meta-analysis comparing the efficacy of RFA and LA, which reported significantly higher volume reduction after RFA (77.8\% [95\% CI: 67.7-88.0]) than after LA $(49.5 \%$ [95\% CI: 26.7-72.4]) (38). We speculate the superior efficacy of RFA to the use of the moving-shot technique, which allows multi-dimensional ablation of the entire tumor safely and effectively $(38,39)$. Interestingly, six of eight included studies of either RFA or MWA applied the moving-shot technique $(14,16,18,20,23,24)$. However, none of the LA studies used it due to the procedure-specific steps in LA, which simultaneously deploys multiple fibers-each with an individual energy emission - in an array around the tumor (40). Our findings were consistent with a previous study by Baek et al., who reported the efficacy of RFA to be slightly superior to that of LA, and that attributed this to the use of the moving-shot technique and an internal cooling system (40). However, our findings were in PTMC and not benign thyroid nodules reported in these studies and that were greater in size $(37,38,40)$.

Overall, thermal ablation techniques were found to be safe in the treatment of PTMC. The pooled proportions of both overall (3.2\% [95\% CI: 1.1-5.2]) and major complications (0.7\% [95\% CI: 0-1.5]) were similar to those previously reported in a meta-analysis evaluating the safety of RFA for the treatment of benign thyroid nodules and recurrent thyroid cancers (pooled proportions of overall and major complications: $2.38 \%$ and $1.35 \%$, respectively) (41). However, in a meta-analysis evaluating the safety and efficacy of MWA for benign thyroid nodules and PTMC, the pooled proportions of major and minor complications were $11.5 \%$ and $5.1 \%$, respectively (42), higher than those reported in this study. Indeed, in the subgroup analysis of safety by treatment modality, we also found MWA to have higher pooled proportions of overall and major complications than the other two modalities, although the difference was not statistically significant. This slight difference may be due to variation in operators' experience or procedural techniques, and thus may not necessarily imply that MWA is safer than the other two

Table 4. Subgroup Analysis of Overall and Major Complications According to the Treatment Modality

\begin{tabular}{|c|c|c|c|c|c|c|}
\hline \multirow[b]{2}{*}{$\begin{array}{l}\text { Treatment } \\
\text { modality }\end{array}$} & \multicolumn{2}{|c|}{ Overall complications } & \multirow[b]{2}{*}{$\mathrm{p}^{\mathrm{b}}$} & \multicolumn{2}{|c|}{ Major complications } & \multirow[b]{2}{*}{$\mathrm{p}^{\mathrm{b}}$} \\
\hline & $\begin{array}{c}\text { Pooled proportions } \\
{[95 \% \text { CI] }}\end{array}$ & $\mathrm{I}^{2 \mathrm{a}}(\%)$ & & $\begin{array}{c}\text { Pooled proportions } \\
{\left[\begin{array}{l}\text { [95\% CI] }\end{array}\right.}\end{array}$ & $\mathrm{I}^{2 \mathrm{a}}(\%)$ & \\
\hline LA & $0.6 \%$ [0-2.5] & 0 & 0.286 & $0 \%$ [0-1.7] & 0 & 0.406 \\
\hline MWA & $5.1 \%[2.8-7.3]$ & 50 & & $2.5 \%[0.9-4.1]$ & 0 & \\
\hline RFA & $2.6 \%[0-5.1]$ & 29 & & $0 \%[0-1.2]$ & 0 & \\
\hline
\end{tabular}

${ }^{\text {a} H i g g i n s ' ~ i n d e x ~ f o r ~ h e t e r o g e n e i t y ~(0-40 \%, ~ m a y ~ h a v e ~ i n s i g n i f i c a n t ~ h e t e r o g e n e i t y ; ~ 30-60 \%, ~ 50-90 \%, ~ a n d ~ 75-100 \% ~ m a y ~ i n d i c a t e ~}$ moderate, substantial, and considerable heterogeneity, respectively).

'Egger's test $(p<0.10$ indicating significant publication bias). 
modalities. Aside from subtle differences in the frequency of complications by treatment modality, no major complications were reported to be life-threatening, confirming that minimal risks are associated with all thermal ablations.

With respect to study heterogeneity, very little heterogeneity was present for the recurrence of PTMC ( $p=0.702$; $\left.I^{2}=0 \%\right)$. However, significant between-study heterogeneity was noted for the complete disappearance of PTMC $\left(p<0.001 ; I^{2}=99 \%\right)$. After subgroup analysis by treatment modality, large heterogeneity was noted for each modality with respect to complete disappearance of PTMC, with $I^{2}$ of more than $90 \%$ for all modalities. We assume that a small number of included studies within each subgroup $(<4$ studies) might be attributable to this finding. Further, significant between-study heterogeneity was noted in the mean volume reduction rate of PTMC $\left(p<0.001 ; I^{2}=86 \%\right)$. The MWA accounted for most of the heterogeneity $\left(I^{2}=92 \%\right)$, whereas LA showed no heterogeneity $\left(I^{2}=0 \%\right)$, possibly suggesting that LA may have more consistent treatment effects than MWA.

All three modalities are based on thermal ablation as the fundamental mechanism of delivering therapeutic effect. The application of localized heating on thyroid tissue creates irreversible cellular damage in LA $(36,43)$, RFA $(40,44)$, and MWA (45). In addition to the widely known benefits of thermal ablations over surgery, such as avoiding surgical scarring and postoperative risks, US-guided delivery of heat specifically to the lesion allows preservation of surrounding normal thyroid tissues and, consequently, of thyroid function. One issue to consider is that lesion-specific targeting may have a risk of overlooking other very tiny microcarcinomas not visible on US; however, the same risk exists for lobectomy when tiny microcarcinomas are in the contralateral lobe (36). Among the included studies, the longest mean followup period was two years, which may not be long enough to confidently determine the efficacy of thermal ablations. Although not included in our data synthesis due to a small sample size (consisting of small papillary thyroid carcinomas not strictly defined as PTMC), a study by Kim et al. included four patients with PTMC who were treated with RFA (46); over a long follow-up period ranging from 36 to 65 months, all four PTMC showed complete disappearance without recurrence. Future studies are needed to further validate the efficacy and safety of thermal ablations with a longer followup period.

There are a few limitations to our study. First, the included studies were regionally concentrated in Asia, and our metaanalytic results may not be similar in other regions where clinical practice settings may differ. Second, the number of included studies was relatively small because treatment of PTMC by thermal ablations was introduced recently, as evidenced from the years of publication of included studies (8 of 11 studies published between 2018 and 2019). In addition, the operators' experiences were not included in the subgroup analysis due to insufficient information and variability of reported data.

In conclusion, the current systematic review and metaanalysis provides a comprehensive review of results on the efficacy and safety of thermal ablation techniques-RFA, MWA, and LA - for the treatment of PTMC. Our results suggest that thermal ablation techniques have reasonable safety and efficacy profiles. However, future research needs to investigate studies with longer follow-up and more heterogeneous populations.

\section{Acknowledgment}

The authors appreciate Dr. Eun Ju Ha for her time and effort in reviewing this article.

\section{Author Disclosure Statement}

No competing financial interests exist.

\section{Funding Information}

No funding was received for this article.

\section{References}

1. Lloyd RV, Buehler D, Khanafshar E 2011 Papillary thyroid carcinoma variants. Head Neck Pathol 5:51-56.

2. Hay ID 2007 Management of patients with low-risk papillary thyroid carcinoma. Endocr Pract 13:521-533.

3. Mazzaferri EL 2007 Management of low-risk differentiated thyroid cancer. Endocr Pract 13:498-512.

4. Haugen BR, Alexander EK, Bible KC, Doherty GM, Mandel SJ, Nikiforov YE, Pacini F, Randolph GW, Sawka AM, Schlumberger M, Schuff KG, Sherman SI, Sosa JA, Steward DL, Tuttle RM, Wartofsky L 20162015 American Thyroid Association Management Guidelines for Adult Patients with Thyroid Nodules and Differentiated Thyroid Cancer: the American Thyroid Association Guidelines Task Force on Thyroid Nodules and Differentiated Thyroid Cancer. Thyroid 26:1-133.

5. Chang YW, Kim HS, Kim HY, Lee JB, Bae JW, Son GS 2016 Should central lymph node dissection be considered for all papillary thyroid microcarcinoma? Asian J Surg 39: 197-201.

6. Spiezia S, Garberoglio R, Milone F, Ramundo V, Caiazzo C, Assanti AP, Deandrea M, Limone PP, Macchia PE, Lombardi G, Colao A, Faggiano A 2009 Thyroid nodules and related symptoms are stably controlled two years after radiofrequency thermal ablation. Thyroid 19:219-225.

7. Huh JY, Baek JH, Choi H, Kim JK, Lee JH 2012 Symptomatic benign thyroid nodules: efficacy of additional radiofrequency ablation treatment session-prospective randomized study. Radiology 263:909-916.

8. Bernardi S, Dobrinja C, Fabris B, Bazzocchi G, Sabato N, Ulcigrai V, Giacca M, Barro E, De Manzini N, Stacul F 2014 Radiofrequency ablation compared to surgery for the treatment of benign thyroid nodules. Int J Endocrinol 2014: 934595.

9. Trimboli P, Castellana M, Sconfienza LM, Virili C, Pescatori LC, Cesareo R, Giorgino F, Negro R, Giovanella L, Mauri G 2019 Efficacy of thermal ablation in benign non-functioning solid thyroid nodule: a systematic review and meta-analysis. Endocrine. DOI:10.1007/s12020-019-02019-3

10. Kim JH, Yoo WS, Park YJ, Park DJ, Yun TJ, Choi SH, Sohn CH, Lee KE, Sung MW, Youn YK, Kim KH, Cho BY 2015 Efficacy and safety of radiofrequency ablation for treatment of locally recurrent thyroid cancers smaller than $2 \mathrm{~cm}$. Radiology 276:909-918.

11. Chung SR, Baek JH, Choi YJ, Lee JH 2019 Longer-term outcomes of radiofrequency ablation for locally recurrent papillary thyroid cancer. Eur Radiol 29:4897-4903.

12. Choi Y, Jung SL, Bae JS, Lee SH, Jung CK, Jang J, Shin NY, Choi HS, Ahn KJ, Kim BS 2019 Comparison of efficacy and 
complications between radiofrequency ablation and repeat surgery in the treatment of locally recurrent thyroid cancers: a single-center propensity score matching study. Int J Hyperthermia 36:359-367.

13. Lim HK, Baek JH, Lee JH, Kim WB, Kim TY, Shong YK, Hong SJ 2015 Efficacy and safety of radiofrequency ablation for treating locoregional recurrence from papillary thyroid cancer. Eur Radiol 25:163-170.

14. Li J, Liu Y, Liu J, Yang P, Hu X, Qian L 2019 A comparative study of short-term efficacy and safety for thyroid micropapillary carcinoma patients after microwave ablation or surgery. Int J Hyperthermia 36:640-646.

15. Ji L, Wu Q, Gu J, Deng X, Zhou W, Fan X, Zhou F 2019 Ultrasound-guided percutaneous laser ablation for papillary thyroid microcarcinoma: a retrospective analysis of 37 patients. Cancer Imaging 19:16.

16. Zhang Y, Zhang MB, Luo YK, Li J, Zhang Y, Tang J 2019 Effect of chronic lymphocytic thyroiditis on the efficacy and safety of ultrasound-guided radiofrequency ablation for papillary thyroid microcarcinoma. Cancer Med 8:5450 5458.

17. Ding M, Tang X, Cui D, Chi J, Shi Y, Wang T, Zhai B, Li P 2019 Clinical outcomes of ultrasound-guided radiofrequency ablation for the treatment of primary papillary thyroid microcarcinoma. Clin Radiol 74:712-717.

18. Teng DK, Li HQ, Sui GQ, Lin YQ, Luo Q, Fu P, Du JR, Jin CX, Wang H 2019 Preliminary report of microwave ablation for the primary papillary thyroid microcarcinoma: a large-cohort of 185 patients feasibility study. Endocrine 64: 109-117.

19. Zhou W, Jiang S, Zhan W, Zhou J, Xu S, Zhang L 2017 Ultrasound-guided percutaneous laser ablation of unifocal T1N0M0 papillary thyroid microcarcinoma: preliminary results. Eur Radiol 27:2934-2940.

20. Jeong SY, Baek JH, Choi YJ, Chung SR, Sung TY, Kim WG, Kim TY, Lee JH 2018 Radiofrequency ablation of primary thyroid carcinoma: efficacy according to the types of thyroid carcinoma. Int J Hyperthermia 34:611-616.

21. Li J, Liu Y, Liu J, Qian L 2018 Ultrasound-guided percutaneous microwave ablation versus surgery for papillary thyroid microcarcinoma. Int J Hyperthermia 34: 653-659.

22. Yue W, Wang S, Yu S, Wang B 2014 Ultrasound-guided percutaneous microwave ablation of solitary T1N0M0 papillary thyroid microcarcinoma: initial experience. Int J Hyperthermia 30:150-157.

23. Teng D, Sui G, Liu C, Wang Y, Xia Y, Wang H 2018 Long-term efficacy of ultrasound-guided low power microwave ablation for the treatment of primary papillary thyroid microcarcinoma: a 3-year follow-up study. J Cancer Res Clin Oncol 144:771-779.

24. Zhang M, Luo Y, Zhang Y, Tang J 2016 Efficacy and safety of ultrasound-guided radiofrequency ablation for treating low-risk papillary thyroid microcarcinoma: a prospective study. Thyroid 26:1581-1587.

25. Zhang L, Zhou W, Zhan W, Peng Y, Jiang S, Xu S 2018 Percutaneous laser ablation of unifocal papillary thyroid microcarcinoma: utility of conventional ultrasound and contrast-enhanced ultrasound in assessing local therapeutic response. World J Surg 42:2476-2484.

26. Liberati A, Altman DG, Tetzlaff J, Mulrow C, Gotzsche PC, Ioannidis JP, Clarke M, Devereaux PJ, Kleijnen J, Moher D 2009 The PRISMA statement for reporting systematic reviews and meta-analyses of studies that evaluate health care interventions: explanation and elaboration. Ann Intern Med 151:W65-W94.

27. Kim SY, Park JE, Lee YJ, Seo H-J, Sheen S-S, Hahn S, Jang B-H, Son H-J 2013 Testing a tool for assessing the risk of bias for nonrandomized studies showed moderate reliability and promising validity. J Clin Epidemiol 66: 408-414.

28. Lewis CA, Allen TE, Burke DR, Cardella JF, Citron SJ, Cole PE, Drooz AT, Drucker EA, Haskal ZJ, Martin LG, Van Moore A, Neithamer CD, Oglevie SB, Rholl KS, Roberts AC, Sacks D, Sanchez O, Venbrux A, Bakal CW 1997 Quality improvement guidelines for central venous access. The Standards of Practice Committee of the Society of Cardiovascular \& Interventional Radiology. J Vasc Interv Radiol 8:475-479.

29. Burke DR, Lewis CA, Cardella JF, Citron SJ, Drooz AT, Haskal ZJ, Husted JW, McCowan TC, Van Moore A, Oglevie SB, Sacks D, Spies JB, Towbin RB, Bakal CW, Society of Interventional Radiology Standards of Practice C 2003 Quality improvement guidelines for percutaneous transhepatic cholangiography and biliary drainage. J Vasc Interv Radiol 14:S243-S246.

30. Kim KW, Lee J, Choi SH, Huh J, Park SH 2015 Systematic review and meta-analysis of studies evaluating diagnostic test accuracy: a practical review for Clinical ResearchersPart I. General Guidance and Tips. Korean J Radiol 16: 1175-1187.

31. Lee J, Kim KW, Choi SH, Huh J, Park SH 2015 Systematic review and meta-analysis of studies evaluating diagnostic test accuracy: a practical review for Clinical ResearchersPart II. Statistical methods of meta-analysis. Korean J Radiol 16:1188-1196.

32. Suh CH, Park SH 2016 Successful publication of systematic review and meta-analysis of studies evaluating diagnostic test accuracy. Korean J Radiol 17:5-6.

33. Higgins JP, Green S 2011 Cochrane Handbook for Systematic Reviews of Interventions. Vol. 4. John Wiley \& Sons, London, UK.

34. Egger M, Davey Smith G, Schneider M, Minder C 1997 Bias in meta-analysis detected by a simple, graphical test. BMJ 315:629-634.

35. Duval S, Tweedie R 2000 Trim and fill: a simple funnelplot-based method of testing and adjusting for publication bias in meta-analysis. Biometrics 56:455-463.

36. Valcavi R, Piana S, Bortolan GS, Lai R, Barbieri V, Negro R 2013 Ultrasound-guided percutaneous laser ablation of papillary thyroid microcarcinoma: a feasibility study on three cases with pathological and immunohistochemical evaluation. Thyroid 23:1578-1582.

37. Korkusuz Y, Gröner D, Raczynski N, Relin O, Kingeter Y, Grünwald F, Happel C 2018 Thermal ablation of thyroid nodules: are radiofrequency ablation, microwave ablation and high intensity focused ultrasound equally safe and effective methods? Eur Radiol 28:929-935.

38. Ha EJ, Baek JH, Kim KW, Pyo J, Lee JH, Baek SH, Dossing $\mathrm{H}$, Hegedus L 2015 Comparative efficacy of radiofrequency and laser ablation for the treatment of benign thyroid nodules: systematic review including traditional pooling and bayesian network meta-analysis. J Clin Endocrinol Metab 100:1903-1911.

39. Baek JH, Kim YS, Lee D, Huh JY, Lee JH 2010 Benign predominantly solid thyroid nodules: prospective study of efficacy of sonographically guided radiofrequency ablation versus control condition. AJR Am J Roentgenol 194:1137-1142. 
40. Baek JH, Lee JH, Valcavi R, Pacella CM, Rhim H, Na DG 2011 Thermal ablation for benign thyroid nodules: radiofrequency and Laser. Korean J Radiol 12:525-540.

41. Chung SR, Suh CH, Baek JH, Park HS, Choi YJ, Lee JH 2017 Safety of radiofrequency ablation of benign thyroid nodules and recurrent thyroid cancers: a systematic review and meta-analysis. Int J Hyperthermia 33:920-930.

42. Cui T, Jin C, Jiao D, Teng D, Sui G 2019 Safety and efficacy of microwave ablation for benign thyroid nodules and papillary thyroid microcarcinomas: a systematic review and meta-analysis. Eur J Radiol 118:58-64.

43. Piana S, Riganti F, Froio E, Andrioli M, Pacella CM, Valcavi R 2012 Pathological findings of thyroid nodules after percutaneous laser ablation: a series of 22 cases with cyto-histological correlation. Endocr Pathol 23:94100.

44. Park HS, Baek JH, Park AW, Chung SR, Choi YJ, Lee JH 2017 Thyroid radiofrequency ablation: updates on innovative devices and techniques. Korean J Radiol 18: 615-623.
45. Heck K, Happel C, Grünwald F, Korkusuz H 2015 Percutaneous microwave ablation of thyroid nodules: effects on thyroid function and antibodies. Int J Hyperthermia 31:560-567.

46. Kim J-H, Baek JH, Sung JY, Min HS, Kim KW, Hah JH, Park DJ, Kim KH, Cho BY, Na DG 2017 Radiofrequency ablation of low-risk small papillary thyroidcarcinoma: preliminary results for patients ineligible for surgery. Int $\mathrm{J}$ Hyperthermia 33:212-219.

Address correspondence to: So-Lyung Jung, MD, PhD Department of Radiology Yeouido St. Mary's Hospital College of Medicine

The Catholic University of Korea Yeongdeungpo-gu 63-ro, 1007345

Seoul

Korea

E-mail: sljung1@catholic.ac.kr 